\title{
Miliary Histoplasmosis Mimicking Miliary Tuberculosis in a Patient Treated with Tumor Necrosis Factor- $\alpha$ Inhibitor Therapy
}

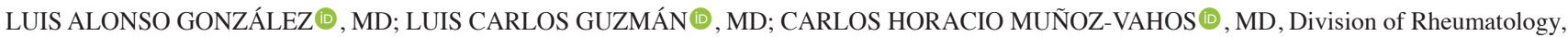
Department of Internal Medicine, School of Medicine, Universidad de Antioquia, Hospital Universitario de San Vicente Fundación, Medellín, Colombia. Address correspondence to Dr. L.A. González, Division of Rheumatology, Department of Internal Medicine, School of Medicine, Universidad de Antioquia, Hospital Universitario de San Vicente Fundación, Calle 64 \#51D-154, Medellín 050010, Colombia. E-mail: luisgona68@ gmail.com. The Hospital Universitario de San Vicente Fundación Research Ethics Committee approved the publication of this report (Act N ${ }^{\circ}$ 26-2019). The patient gave written informed consent to publish the material. J Rheumatol 2020;47:1450-51; doi:10.3899/jrheum.191158
\end{abstract}

In patients receiving anti-tumor necrosis factor (TNF)- $\alpha$ agents, a miliary pattern on chest imaging is often attributed to tuberculosis. However, fungal infections (histoplasmosis, blastomycosis, and coccidioidomycosis) and metastatic pulmonary disease should also be considered ${ }^{1,2}$.

A 40-year-old woman, diagnosed with ankylosing spondylitis, was in remission with adalimumab. She presented with a 3-week history of fever, night sweats, dyspnea, and dry cough. She reported exposure to demolition dust from a building adjacent to her workplace. On admission, she was normotensive and tachycardic, and oxygen saturation was $96 \%$ with supplemental oxygen $(2 \mathrm{l} / \mathrm{min})$. Breath sounds were decreased bilaterally. Chest radiograph and high-resolution computed tomography showed widespread miliary nodules throughout both lungs (Figure 1). Three sputum acid-fast bacillus (AFB) smears were negative. On the bronchoalveolar lavage sample, AFB stain, AFB culture, PCR

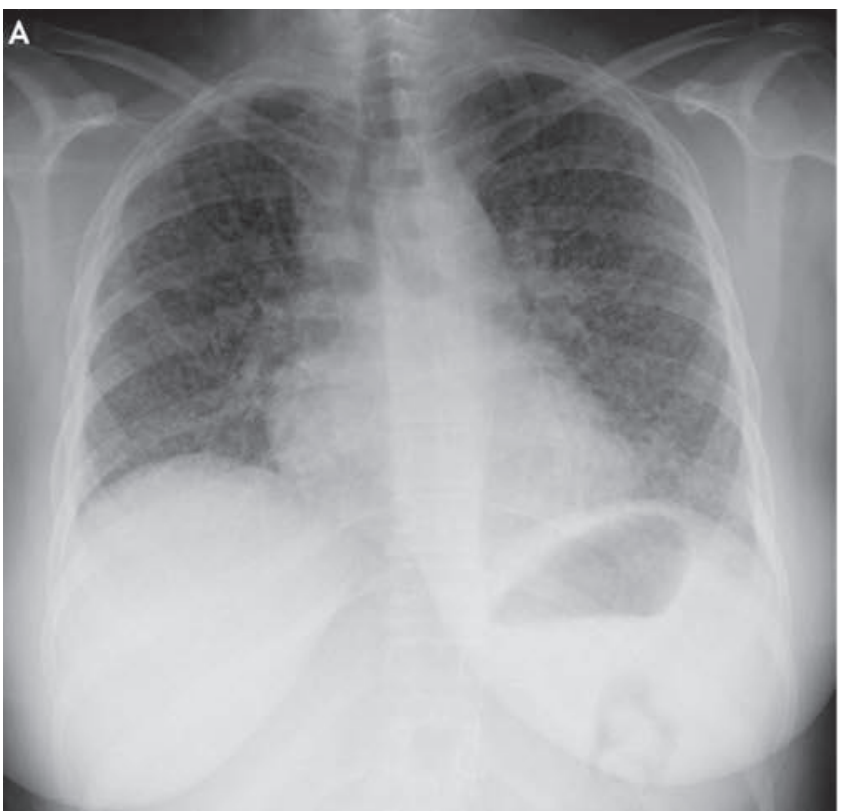

for Mycobacterium tuberculosis complex were all negative, while fungal culture was positive for Histoplasma capsulatum. Transbronchial biopsy identified non-caseating granulomas (Figure 2A) and small ovoid or pear-shaped yeast with narrow-based budding consistent with $H$. capsulatum (Figure 2B). Urine Histoplasma galactomannan antigen was positive. Amphotericin-B was administered for 2 weeks followed by 12 months of oral itraconazole, with marked clinical improvement after therapy.

Histoplasmosis is the most frequent invasive fungal infection complicating anti-TNF- $\alpha$ therapy ${ }^{3}$; however, miliary histoplasmosis is a rare clinical presentation that mimics miliary tuberculosis, and diagnostic delays may lead to increased mortality ${ }^{3}$. Anti-TNF- $\alpha$ therapy discontinuation is recommended and antifungal treatment must be given for at least 12 months. Resumption of anti-TNF- $\alpha$ appears safe if antifungal therapy is administered for 12 months $^{4}$.

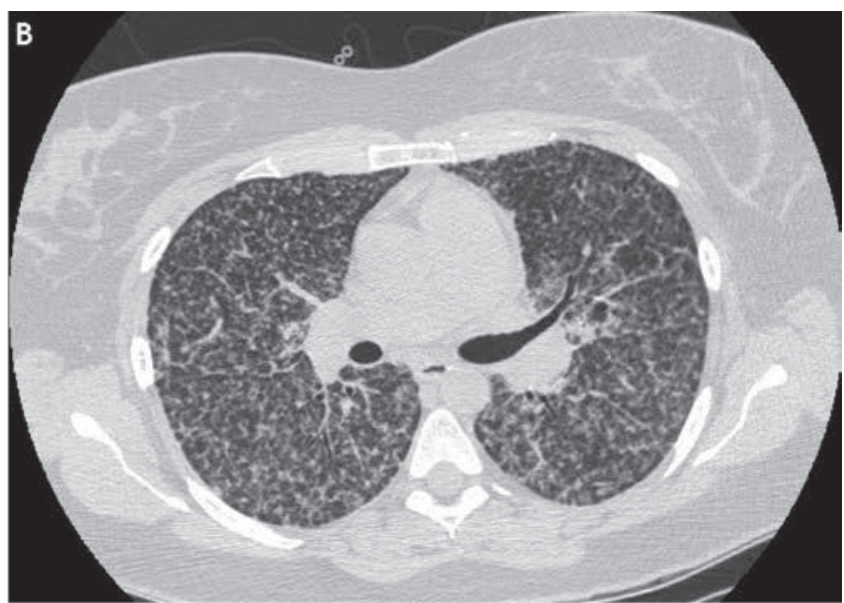

Figure 1. A. Chest radiograph demonstrates diffuse bilateral pulmonary micronodules in a miliary pattern. B. High-resolution computed tomography shows miliary nodules throughout both lung fields.

Personal non-commercial use only. The Journal of Rheumatology Copyright $@$ C 2020. All rights reserved. 

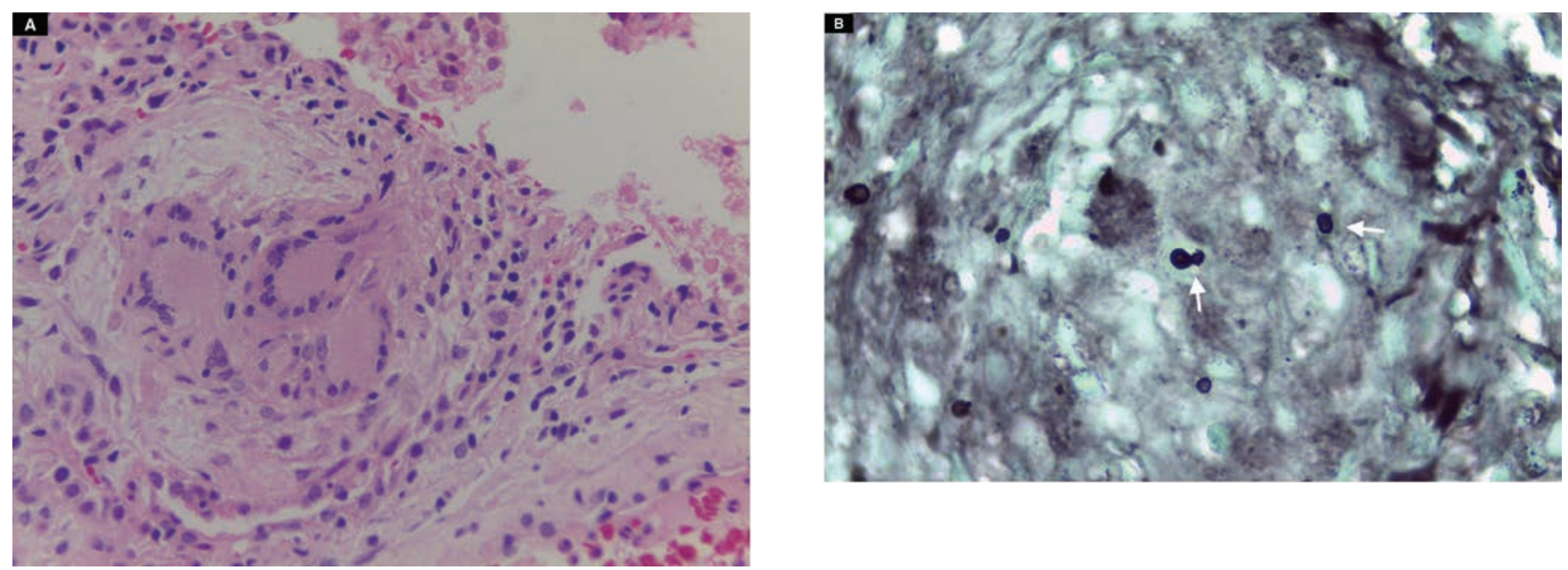

Figure 2. A. Pathology from transbronchial biopsy showing non-caseating granuloma with multinucleated giant cells (H\&E, $\times 40)$. B. Pathology from transbronchial biopsy showing small black ovoid and budding yeasts (white arrows) consistent with Histoplasma capsulatum (Gomori's methenamine silver, $\times 100$ ).

\section{REFERENCES}

1. Lum J, Abidi MZ, McCollister B, Henao-Martínez AF. Miliary histoplasmosis in a patient with rheumatoid arthritis. Case Rep Med 2018;2018:2723489.

2. Haroon M, Cushen B, Veale D. Miliary nodularity in a patient receiving TNF inhibitors is not always miliary tuberculosis. J Rheumatol 2012;39:651-2.
3. Hage CA, Bowyer S, Tarvin SE, Helper D, Kleiman MB, Wheat LJ. Recognition, diagnosis, and treatment of histoplasmosis complicating tumor necrosis factor blocker therapy. Clin Infect Dis 2010;50:85-92.

4. Vergidis P, Avery RK, Wheat LJ, Dotson JL, Assi MA, Antoun $\mathrm{SA}$, et al. Histoplasmosis complicating tumor necrosis factor- $\alpha$ blocker therapy: a retrospective analysis of 98 cases. Clin Infect Dis 2015;61:409-17. 